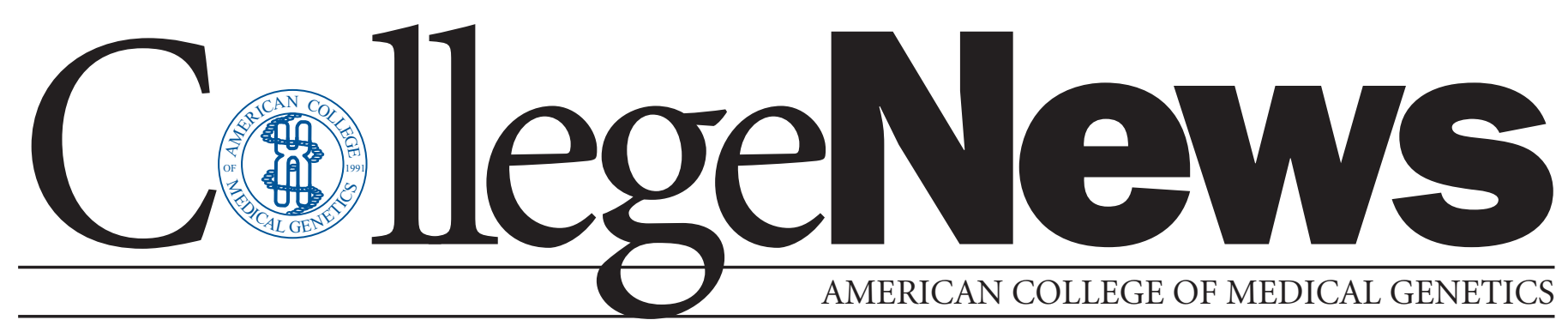

\title{
Announcements
}

\section{Costello Syndrome}

Costello Syndrome is a rare genetic disorder, with about 150 people identified worldwide since it was first recognized as a distinct syndrome in 1971. It is suspected to be the result of an autosomal dominant gene. Individuals with Costello Syndrome typically exhibit 3 clinical phases: (1) polyhydramnios during pregnancy, and increased birth weight; (2) "coarse" facial features and hypotonia that may lead to a suspicion of a storage disorder in infancy through early childhood. There is loose skin, lax joints, dramatic weight loss, failure to thrive, global developmental delay, and general medical fragility. (3) In late childhood, nutrition improves, and the children are generally more medically stable. Distinctive skin findings include papillomata, hyperkeratosis, and pigmentation. Other problems include heart anomalies (a broad spectrum from mild arrhythmia to severe hypertrophic cardiomyopathy), malignant tumors (especially rhabdomyosarcoma), and orthopedic issues. Efforts are being made in the US and UK to understand the clinical features of Costello syndrome, particularly its relationship to cancer.

At present, diagnosis is clinical. Storage disorders must be ruled out. Children diagnosed with Noonan and Cardio-FacioCutaneous Syndrome have been rediagnosed with Costello Syndrome, and vice-versa. Although there are similarities to other syndromes, Costello syndrome has a distinctive appearance, which can be recognized in our children's faces. Both the CostelloKids Web site and the brochure have multiple images of the children.

If you suspect one of your patients may have Costello Syndrome, we have several ways to help. We have a Web site, http:// www.costellokids.org.uk/; or you can contact the Costello Program at the duPont Hospital for Children, http://www.nemours.org/ no/aidhc/svcs/div2626.html. Brochures are available and can be ordered through the CostelloKids Web site. We welcome your participation.

\section{Kidney disease}

Kidney disease? Call NIH at 1-800-411-1222 for information on new kidney studies, including Lupus Nephritis, Membranous Nephropathy, and Focal Segmental Glomerulosclerosis. Treatment provided at no cost. Transportation may be provided. (TTY: 1-866-411-1010) E-mail prpl@cc.nih.gov. NIH is part of Department of Health and Human Services. 


\section{Diplomates Certified In 2002- 2003}

ABMG is proud to acknowledge the individuals who achieved certification in 2002-2003:

\section{Clinical Geneticists}

Adams, Darius

Al-Owain, Mohammed A.

Al-Sanna'a, Nouriya A.

Bashford, Michael T

Biggio Jr, Joseph R

Bober, Michael B.

Chung, Wendy Kay

Colby, Randall Stuart

Corzo, Deyanira

Cowan, Linda S.

Dar, Peer

Diaz, George A.

Dipple, Katrina M.

Eash, Delaina D.

Garganta, Cheryl L.

Golomb, Mayana

Gordon, Ora B.

Hamid, Rizwan

Hand, Jennifer L.

Hedera, Peter

Holmes, Rebecca Johnson

Hoover-Fong, Julie Elizabeth

Ibrahim, Jennifer

Introne, Wendy J.

Jari, Shama Dhandha

Keegan, Catherine E.

Kim, Sook Za

Lacbawan, Felicitas L.

Lalani, Seema R.

Lee, Grace Y.

Levy, Paul Arthur

Lichter-Konecki, Uta

Lin, Ruth J.

Lyon, Helen M.

Manning, Melanie A.

Martin, Donna M.

McBride, Kim Lewis

Miller, Daniel G.

Mitchell, Anna L.

Moghaddam, Billur

Morelli, Susan Horst

Murray, Michael F.

Northrop, Jennifer L.

Olney, Richard S.

Olson, Rick L.

Pal, Tuya

Picker, Jonathan D.

Pinter, Robert A.

Plotner, Pamela L.

Rajkovic, Aleksandar

Rastogi, Amit

Rauen, Katherine A.
Sampson, Jone Elizabeth

Schweitzer, Daniela N.

Slavotinek, Anne M.

Smith, Wendy Ellyn

Soper, Robert James

Souter, Vivienne L.

Stanley-Christian, Heather K.

Tahmaz, Fatma E.

Taylor, Matthew R.G.

Tegay, David Harrison

Venditti, Charles Paul

Wang, Tao

Wang, Xia

Ware, Stephanie M

Wattendorf, Daniel J.

Zhang, Hui

\section{PhD Medical Geneticist}

Neiswanger, Katherine

\section{Clinical Biochemical Geneticists}

Al Hassnan, Zuhair N.

Al-Owain, Mohammed A.

Dasouki, Majed J.

Dipple, Katrina M.

Ficicioglu, Can $\mathrm{H}$.

Garcia-Soto, Elisdel M.

Garganta, Cheryl L.

Goh, Denise L.

Ierardi-Curto, Lynne A.

Introne, Wendy J.

Kim, Sook Za

Mardach, Rebecca

Matern, Dietrich

Phornphutkul, Chanika

Salazar, Denise

Schroer, Richard J.

Strovel, Erin Thompson

Sutton, Vernon Reid

Tang, Yingying

Tsai, Chun-Hui

Venditti, Charles Paul

Woontner, Michael

\section{Clinical Cytogeneticists}

Bao, Liming

Batish, Sat Dev

Boles, Debra

Boyar, Fatih Ziya

Call, Linda M.

Dave, Bhavana J.

Dennis, Thomas R.

Dolan, Michelle

Fang, Min

Gupta, Shiphali

Iyer, Ramaswamy K.

Ketterling, Rhett Patrick
Lee, Charles

Levy, Brynn

Li, Peining

Morrissette, Jennifer J.D.

Phillips, Karen K.

Sago, Haruhiko

Sasi, Ramakrishnan

Smolarek, Teresa Anne

Sreekantaiah, Chandrika

Tantravahi, Umadevi

Tonk, Vijay S.

Wilson, Kathleen S.

Wiltshire, Rodney

Wolf, Nancy G.

Yenamandra, Aswani K.

\section{Clinical Molecular Geneticists}

Al Hassnan, Zuhair N.

Bashford, Michael T.

Batish, Sat Dev

Bober, Michael B.

Booker, Jessica K.

Bowles, Karla R.

Buller, Arlene M.

Buyse, Inge $\mathrm{M}$.

Cai, Li

Christiano, Angela M.

Demirci, F. Yesim

Dolan, Michelle

Dong, Jianli

Dong, Liang

Edelmann, Lisa J.

Fang, Min

Fang, Ping

Friedman, Kenneth J.

Friez, Michael J.

Goonewardena, Ponmani

Hibbard, Michele K.

Howe, John

Johnston, Jennifer Jill

Koty, Patrick P.

Limprasert, Pornprot

Mao, Rong

McVie-Wylie, Alison J.

Morrissette, Jennifer J.D.

Nicklas, Janice A.

Parks, Sharie B.

Peng, Mei

Sanoudou, Despina

Saunders, Carol

Schwarze, Ulrike

Shabbeer, Junaid

Verselis, Sigitas J

Wan, Minghong

Wang, XinJing

Wood, Timothy C.

$\mathrm{Wu}$, Bai-Lin

Xiao, Sheng

Zariwala, Maimoona B.A.H. 